\title{
Estudo preliminar da aplicação da teoria de dutos atmosféricos às linhas de instabilidade amazônicas
}

\author{
Thermodynamic analysis of days with and without formation of amazon squall \\ lines though of the atmospheric ducting theory
}

\author{
Clênia Rodrigues Alcântara
}

Universidade Federal de Campina Grande - UFCG, Campina Grande, PB, Brasil

\begin{abstract}
Resumo
Este trabalho apresenta uma análise de perfis do vento e de temperatura potencial e temperatura potencial equivalente em sítios localizados próximos à cidade de Belém - PA às 00, 06, 12 e 18 UTC nos dias 20,23, 24 e 25 de junho de 2011, sendo os dias 20 e 25 aqueles em que não foram observadas a formação de linhas de instabilidade (LI), e os dias 23 e 24, dias com a formação desses sistemas. O intuito é averiguar características termodinâmicas que diferencie dias com e sem LI e se a teoria de dutos atmosféricos pode ser aplicada para explicar o papel dos jatos de baixos níveis no processo de formação e propagação destas. Como resultados, obteve-se que o perfil de vento continua sendo elemento aparentemente decisivo e com grande contribuição para o processo já, que para os perfis de temperatura potencial, potencial equivalente e potencial equivalente de saturação não foi perceptível diferenças entre os dias com e sem LI. A maioria das características necessárias para a formação dos dutos atmosféricos, como camadas estáveis estaticamente, profundidade adequada e um nível crítico, foram encontradas nos perfis estudados.
\end{abstract}

Palavras-chave: Linhas de Instabilidade, Amazônia, Termodinâmica, Teoria de dutos atmosféricos

\begin{abstract}
This paper presents an analysis of the profiles of wind and potential temperature and equivalent potential temperature at sites located near Belem City in Pará State at 00, 06, 12 and 18 UTC on June 20,23, 24 and 25 of 2011. The days 20 and 25 are those in which were not observed the formation of squall lines (SL) and days 23 and 24 are those with formation of these systems. The aim of this work is to determine a thermodynamic and dynamic characteristic that distinguishes days with and without SL and the theory of atmospheric ducts can be applied to explain the role of low level jets in the process of formation and propagation of these systems. As a result, it was found that the wind profile remains crucial element and with great contribution to the process. Differences in potential and potential equivalent temperature profiles were not noticeable between days with and without SL. Most of the features necessary for the formation of atmospheric duct layers, as statically stable, adequate depth and a critical level, were found in the atmospheric profiles studied here.
\end{abstract}

Keywords: Squall lines, Amazonia, Thermodynamics, Atmospheric Ducting theory 


\section{Introdução}

As Linhas de instabilidade da Amazônia (LIA) são sistemas de grande importância para a região, por serem um dos mais importantes produtores de chuva, e para a circulação global, devido ao seu eficiente transporte de umidade, massa e gases para a alta troposfera. Elas podem se formar por toda a bacia, mas aquelas que se formam na costa norte do Brasil são as mais interessantes, por conseguirem se deslocar até 3000 km, chegando próximo a Cordilheira dos Andes, e com ciclo de vida que pode chegar a $48 \mathrm{~h}$.

De acordo com Kousky (1980), uma vez que um cumulonimbus se desenvolve ao longo da frente de brisa, a linha de convecção ativa pode continuar a se propagar para o interior do continente, possivelmente, como uma linha de instabilidade. Sun e Orslanski (1981) também avaliaram o mecanismo de propagação das linhas de instabilidade tropicais usando um modelo linear no qual a circulação de brisa produziu uma onda propagante associada com a instabilidade trapezoidal, que é devido a oscilação diurna do perfil de temperatura da camada limite.

Cohen et al. (1995) documentaram as condições ambientais associadas com esses sistemas convectivos nessa região, por ocasião do ABLE 2B. Verificaram que o vento zonal médio máximo ocorreu em torno de 800 hPa em todos os casos e que havia uma camada contendo velocidade constante e que esta camada variava entre as diferentes situações estudadas. Alcântara et al. (2011) fizeram uma climatologia com 10 anos de reanálises e confirmaram o que já foi observado quanto a existência do jato em baixos níveis (JBN). Além disso, verificaram que para LI propagantes a profundidade destes jatos era maior, chegando aos médios níveis.

Cohen (1996) utilizou simulações numéricas para verificar um caso de linha de instabilidade que se formou na costa norte do Brasil e verificou que nesta simulação havia padrões de ondas de gravidade se propagando junto com o sistema. Constatou que as ondas associadas com as linhas de instabilidade da Amazônia tinham comprimento vertical entre 6 e 10 $\mathrm{km}$, comprimento horizontal entre $200-250 \mathrm{~km}$ e velocidade em torno de 10 m.s-1.

Warner et al. (2003) desenvolveu uma simulação para verificar padrões diurnos da precipitação no noroeste da América do Sul e verificaram que uma linha de instabilidade amazônica foi simulada. No caso, a propagação da LI não foi simplesmente pela dinâmica da piscina fria. A propagação pareceu envolver o jato de leste próximo a $650 \mathrm{hPa}$, que advectou anomalias de umidade nos médios níveis. Segundo eles, as ondas de gravidade, movendo-se para oeste com velocidade de fase menor que a velocidade dos jatos de leste, pareceram estar aprisionadas nos baixos níveis. Então, se estas ondas induzem convecção, cujo aquecimento por sua vez reforça a onda, uma banda de chuva propagante pode surgir e se propagar por longas distâncias.

Assim, uma questão surge: qual mecanismo permite que estes sistemas se propagem por tão longas distâncias sem se dissiparem? Uma possível explicação pode ser a teoria dos dutos atmosféricos descrita por Lindzen e Tung (1976) [LT]. Eles determinaram as condições necessárias para a atmosfera suportar ondas propagando-se em uma camada sem perder energia.

Ralph et al. (1993) observaram um evento ligado a ondas de gravidade no sudoeste da França no verão de 1984 e constataram que a onda se propagou em um duto atmosférico. A estrutura da estabilidade estática foi favorável a existência do duto. Um nível crítico estava presente dentro ou apenas acima da camada de estabilidade estática baixa que tampou o duto. A estrutura vertical observada foi aquela de uma onda estável, com o topo da camada estável correspondendo a um antimodo e a metade do comprimento vertical da onda abaixo do nível crítico. A onda foi observada quando havia a existência de atividade extensiva associada com duas linhas de convecção profunda na região.

Chun e Lin (1995) investigaram a resposta da atmosfera a uma linha de instabilidade, representada por uma piscina fria induzida por resfriamento evaporativo. Resolveram analiticamente perturbações lineares bi-dimensionais forçadas pelo resfriamento diabático na presença de um nível crítico. O escoamento do ambiente foi assumido ter uma estrutura de três camadas da mesma forma que Lindzen e Tung (1976), excerto que um resfriamento diabático foi especificado na camada mais baixa. Assumiram que a camada com o nível crítico pode ser estável ou instável dinamicamente. Como resultados, obtiveram que quando a camada é dinamicamente estável, quase toda a energia da onda é absorvida próximo ao nível critico. Para o caso instável, as ondas podem ser parcialmente ou totalmente refletidas pelo nível crítico dependendo do número de Richardson. Ondas podem ser totalmente refletidas ou transmitidas, simultaneamente, à medida que viajam através do nível crítico no processo de extração de energia do escoamento médio com cisalhamento. Usando as condições propostas por Lindzen e Tung (1976), encontraram que a magnitude da perturbação da velocidade vertical foi 6 vezes maior que para um caso com a presença de duto, quando comparada a um caso sem, e com a mesma taxa de resfriamento.

Monserrat e Thorpe (1996) tentaram explicar as propriedades de um evento de onda de gravidade observado em Mallorca usando um conjunto de microbarográfos. As ondas se propagaram por longas 
distâncias com uma velocidade em torno de 29 m.s-1 e foram não-dissipativas. Mostraram que quando o cisalhamento do vento está presente no duto, o número de modos é claramente reduzido pela absorção da onda no nível crítico. Então, o cisalhamento fornece um mecanismo pelo qual o espectro dos modos das ondas de gravidade no duto é substancialmente limitado.

Após uma longa pesquisa bibliográfica, se constatou que não há muitos trabalhos na literatura que demonstrem a aplicação da teoria de dutos atmosféricos descrita por Lindzen e Tung (1976). Uma das possíveis explicações é que para sua demonstração é necessário um conjunto de dados bastante rico em informações e com resolução espacial e temporal alta, o que é bastante difícil de obter. Em relação às linhas de instabilidade da Amazônia, também foi possível perceber que não há trabalhos na literatura que mostrem quantitativamente a existência dos dutos atmosféricos. Apenas algumas poucas indicações de que este pode ser um dos mecanismos responsáveis pela propagação destes sistemas.

Este trabalho tem como objetivo detalhar e testar as condições para a formação dos dutos atmosféricos descritas por LT em dias com e sem a ocorrência de linhas de instabilidade da Amazônia. Além disso, os perfis atmosféricos serão analisados termodinamicamente a fim de se obter mais informações a respeito do ambiente de formação das LI.

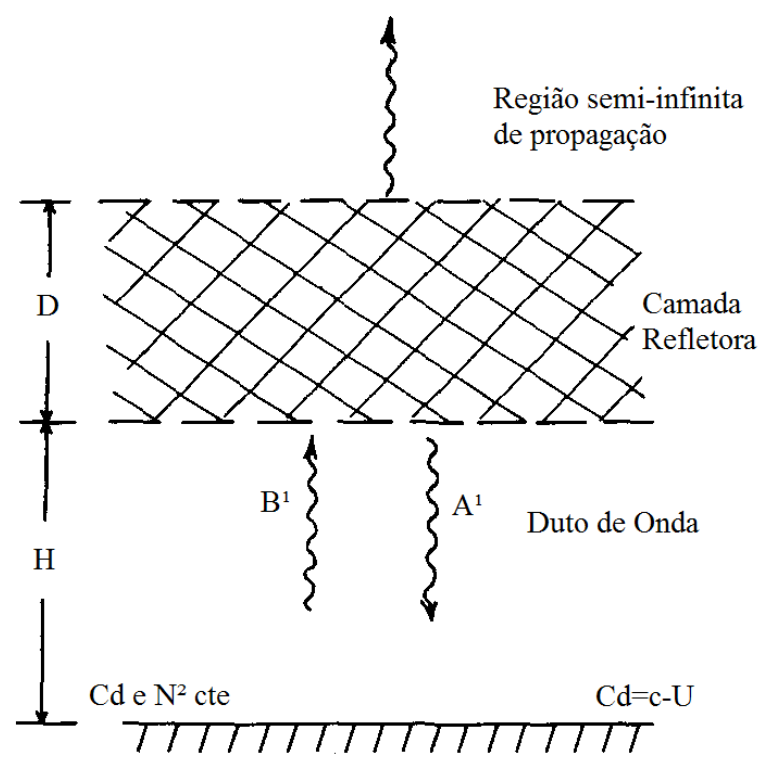

Figura 1 - Diagrama esquemático da atmosfera-modelo (adaptado de Lindzen e Tung, 1976).

\section{Materiais e Métodos}

\subsection{Teoria dos dutos atmosféricos}

Atividades de cúmulos são frequentemente organizadas em estruturas de bandas que são carac- terizadas por comprimento de 100 a $400 \mathrm{~km}$ e viajam relativas ao escoamento médio com velocidade de fase de 10 a 30 m.s-1 [Severe Environmetal Storms and Mesoscale Experiment Project Development Plan - (SESAME), 1974].

Em algumas situações, grande parte da troposfera é potencialmente instável e o ar à superfície é quente e úmido, como nos casos tropicais. Assim, o calor latente liberado nos cumulonimbus parece ser capaz de forçar as ondas que em troca organizam a convecção (wave - CISK). Há outros casos nos quais a convecção fica restrita a níveis mais altos, pouca precipitação e uma troposfera estável em baixos níveis. O problema nesses casos é que ondas de comprimento e velocidade de fase observados podem se propagar livremente na vertical e, por isso, na ausência do forçamento térmico fornecido pela CISK, perderiam sua energia.

Lindzen e Tung (1976) encontraram que ondas podem se propagar por mais tempo na horizontal se os níveis mais baixos da troposfera forem estáveis e limitados por uma região acima que reflita efetivamente as ondas que se propagam na vertical. Cria-se então, nessa região, um duto onde essas ondas que se propagam horizontalmente não perdem grande quantidade de energia e, assim, não precisam de forçamento energético. Esses autores investigaram sob quais circunstâncias as ondas de mesoescala podem existir e determinaram o que poderia fornecer uma boa superfície refletora.

Assim, a atmosfera foi representada por 3 camadas, sendo uma a do duto propriamente dito, uma camada refletora e uma região semi-infinita de propagação no topo. A Figura 1 é a represemtação gráfica deste modelo.

Logo, as propriedades básicas necessárias para a existência de dutos atmosféricos, segundo LT, são:

1. Uma camada estável estaticamente $\left(\mathrm{N}^{2}>0\right)$ para que as ondas possam se propagar;

2. O duto deve ter espessura tal que acomode um quarto do comprimento vertical da onda e que, por sua vez, relaciona-se a velocidade de fase observada;

3. No topo da camada estável deve haver um bom refletor (85\%). Se o refletor é fraco, a energia da onda será rapidamente perdida;

4. A velocidade do vento no duto não pode ser igual à velocidade de fase da onda. Se isso ocorrer, um nível crítico pode estar situado em uma região estável com $\mathrm{Ri}>1 / 4$ e as ondas seriam totalmente absorvidas;

Para a camada refletora, existem três condições:

1. O número de Richardson tem de ser menor que 0,25 ;

2. A camada deve ser potencialmente instável; 
3. Dentro ou acima pode haver um nível em que a velocidade do escoamento e a velocidade das ondas sejam iguais. É o chamado nível crítico;

Estas condições serão testadas em perfis atmosféricos para dias com e sem a ocorrência de linhas de instabilidade costeiras na Amazônia. A seção a seguir descreve estes dados e os procedimentos utilizados não só para análise de dutos como também para a análise termodinâmica e dos perfis de vento.

\subsection{Dados e procedimentos metodológicos}

Para cumprir com os objetivos propostos neste trabalho, foram analisados casos de linhas de instabilidade da Amazônia ocorridos no período entre 01 e 30 de junho de 2011. Este período corresponde a uma das campanhas do projeto CHUVA (Cloud processes of tHe main precipitation systems in Brazil:A contribUtion to cloud resolVing modeling). O projeto CHUVA teve como objetivos reduzir as incertezas na estimativa da precipitação e progredir no conhecimento dos processos das nuvens, principalmente, das nuvens quentes. Desta forma, foram realizados experimentos de campo em sete sítios com diferentes padrões climáticos, para estudar os regimes de precipitação no Brasil. Esses experimentos utilizaram radar polarimétrico, lidar, radiômetro de microonda, disdrômetros, radiosondas e vários outros instrumentos. As análises, nestes sítios, foram realizadas considerando as características microfísicas e a evolução com o ciclo de vida, os modelos de estimativa de precipitação, o desenvolvimento das tempestades e a formação de descargas elétricas, os processos na camada limite e a modelagem da microfísica (Battaglia et al., 2011).

Foram utilizadas sondagens atmosféricas realizadas em torno da cidade de Belém, em dois sítios chamados São Miguel do Guamá $\left(1,62^{\circ} \mathrm{S} ; 47,48^{\circ} \mathrm{W}\right)$ e Tomé Açu $\left(2,41^{\circ} \mathrm{S} ; 48,23^{\circ} \mathrm{W}\right)$. Os dias analisados foram 20, 23 e 25 de junho nos horários das 00, 06, 12 e 18 UTC para o sítio Tomé Açu e 24 de junho, nos mesmos horários, para São Miguel do Guamá, já que a sondagem do dia 24 em Tomé Açu possui falhas que inviabilizam as análises. Mesmo assim, o dia 24 não possui sondagem para as 18 UTC. Os dias 23 e 24 de junho correspondem aqueles em que se observou dois casos de LI e os dias 20 e 25 de junho, não houveram casos de LI.

Estas sondagens serão analisadas do ponto de vista cinemático, através do perfil de vento, e termodinâmico, através dos perfis de temperatura potencial $(\theta)$, temperatura potencial equivalente $(\theta \mathrm{e}) \mathrm{e}$ temperatura potencial equivalente de saturação ( $\theta$ es). As sondagens de vento que fornecem informações sobre intensidade e direção foram decompostas em suas componentes zonais e meridionais.
Estas informações complementares serão muito úteis no entendimento de características associadas às linhas de instabilidade da Amazônia e, também, servem de base para a análise da existência de dutos atmosféricos que se pretende desenvolver aqui.

Inicialmente, identificaram-se nos perfis de $\theta \mathrm{e}$, as camadas com instabilidade potencial . $\left(\frac{\partial \theta_{e}}{\partial z}<0\right)$

Após identificadas estas camadas, foram calculados os números de Richardson correspondentes, segundo LT:

$$
R i=\frac{N^{2} \Delta Z^{2}}{(2 \bar{U})^{2}}
$$

em que $\mathrm{N}^{2}$ é a frequência de Brunt-Vaisalla, $\triangle Z$ é a espessura da camada e $\bar{U}$ é a velocidade média da camada. Estes parâmetros foram calculados como se segue:

$$
\begin{gathered}
N^{2}=\frac{g}{\Delta Z} \ln \left(\frac{\theta_{\text {Topo }}}{\theta_{\text {Base }}}\right) \\
\Delta Z=H \ln \left(\frac{p_{\text {base }}}{p_{\text {topo }}}\right)
\end{gathered}
$$

sendo $H=\frac{R \bar{T}}{g} \quad$ é a escala de altitude, R é a constante dos gases para o ar seco, $\bar{T}$ é a temperatura média da camada e g é a gravidade.

Quando a camada apresentou $\mathrm{Ri}<0,25$, ou seja, atendeu uma das exigências para ser camada refletora, foi calculada a espessura da camada $(\Delta Z)$ logo abaixo, sua estabilidade estática e seu número de Richardson (Ri) utilizando as mesmas fórmulas mostradas acima. Desta forma, também se verificou se esta camada atende os requisitos para ser a camada do duto propriamente dito.

Também foi preciso inferir as características das ondas de gravidades (velocidade de fase na vertical, $\mathrm{m}$, e velocidade de fase zonal, k) que possivelmente estão sendo aprisionadas na região de formação das LI. Para isto, foram utilizadas as formulações básicas do Holton (2004) e são mostradas a seguir:

$$
m=\frac{2 \pi}{L_{z}} ; k=\frac{2 \pi}{L_{x}} ; \hat{v}=v-\bar{u} k= \pm \frac{N k}{\left(k^{2}+m^{2}\right)^{1 / 2}} ; c_{x}=\frac{\hat{v}}{k}
$$

em que Lz e Lx são os comprimentos das ondas na vertical e na horizontal, $\hat{v}$ é a frequência intrínseca, $\mathrm{N}^{2}$ é a freqüência de Brunt-Vaisalla e cx é a velocidade de fase das ondas. 


\section{Resultados e Discussão}

\subsection{Perfis de vento}

Os perfis de vento para os dias com e sem linha de instabilidade foram analisados e se encontram nas Figuras 2 e 3. Para isso, o vento foi decomposto em suas componentes zonal (u) e meridional (v).

Observando a componente zonal do vento, percebe-se que para o dia 20 (sem LI), o JBN das 12 UTC foi o mais intenso e abaixo de $900 \mathrm{hPa}$. O perfil das 06 UTC também mostrou JBN, porém com 8 m.s-1. Os demais horários não mostraram JBN definidos. $\mathrm{O}$ dia 25 (também sem LI) apresentou JBN às 00, 06 e 12 UTC sendo suas intensidades 14, 12 e 10 m.s-1, respec- tivamente. O JBN manteve-se em torno de $900 \mathrm{hPa}$. Às 18 UTC não se observou jato em baixos níveis. Em 23 de junho (dia com LI), o único horário que apresentou JBN foi às 12 UTC com intensidade de 13 m.s-1 e em torno de $800 \mathrm{hPa}$. Por fim, o dia 24 (também com LI) apresentou JBN nos 3 horários disponíveis, porém o das 12 UTC foi o mais intenso com quase $14 \mathrm{~ms} 1 \mathrm{de}$ intensidade. Esses máximos também ocorreram em torno de $900 \mathrm{hPa}$.

Como já indicado por Alcântara et al. (2011), Cohen et al. (1995) e Silva Dias e Ferreira (1992), o horário das 12 UTC parece ser o mais importante em se tratando da formação de linhas de instabilidade da Amazônia. Ele corresponde às 09 h da manhã no (a)

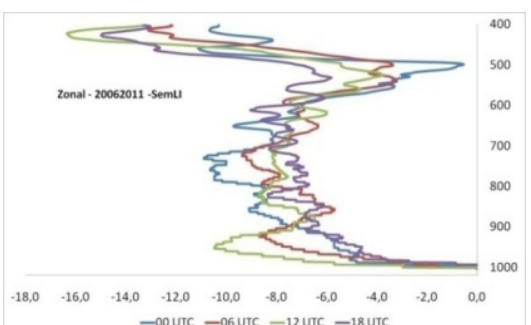

(c)

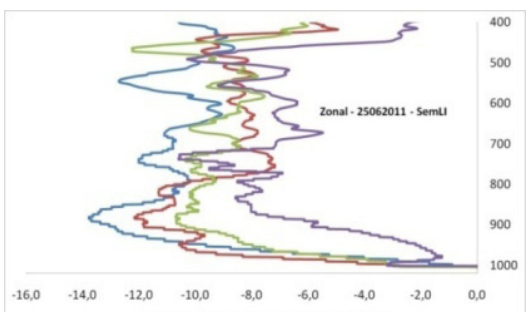

(e)

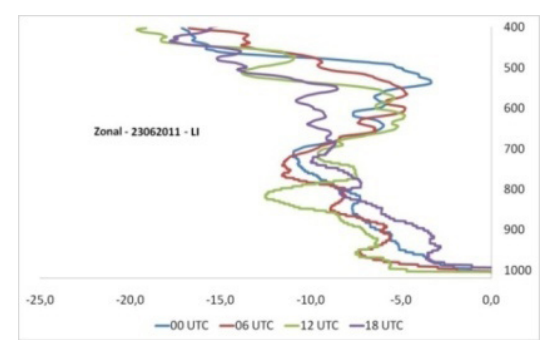

(g)

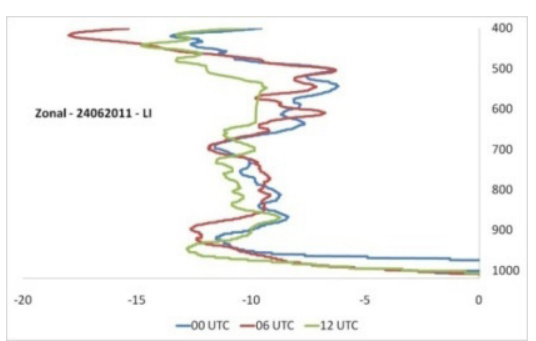

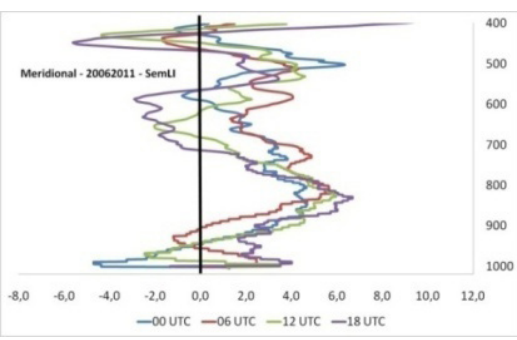

(b)

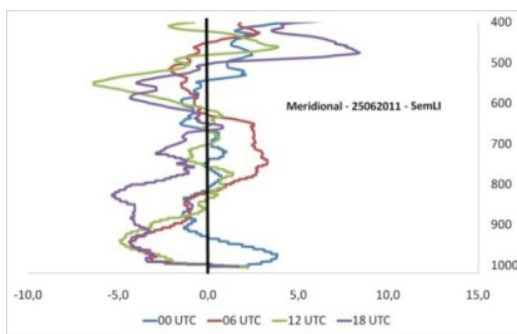

(d)
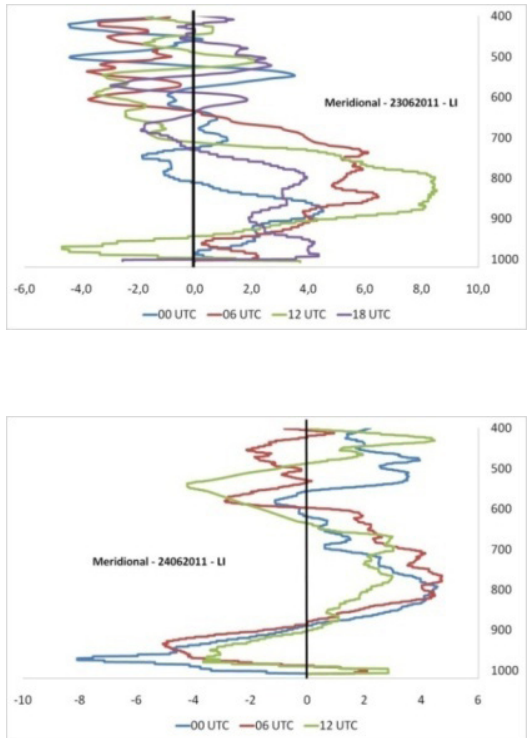

(f)

(h)

Figura 2 - Perfis das componentes zonal (a, c, e e g) e meridional (b, d, f e h) do vento para os dias 20 (sem LI), 25 (sem LI), 23 (com LI) e 24 (com LI) de junho de 2010 às 00 (linha azul), 06 (linha vermelha), 12 (linha verde) e 18 UTC (linha roxa). 
horário local e é quando a circulação de brisa marítima começa a se desenvolver. Os horários da 00 e 06 UTC ainda correspondem ao período noturno e às 18 UTC corresponde a um horário em que, mesmo a brisa nos dias em que não houve a formação de LI, já se desenvolveu e, na maioria dos casos, já se deslocou continente adentro.

Os perfis da componente zonal mostrados aqui corroboram com o que é apontado na literatura quanto à intensidade dos jatos de baixos níveis e sua localização, pois apresentaram 13-14 m.s-1 e estiveram entre 800 e $900 \mathrm{hPa}$. Às 00 UTC até aparece JBN, porém é menos definido. Às 06 UTC, não se observa um padrão definido entre os dias com e sem formação de LI. Às 12 UTC é o horário em que eles aparecem melhor definidos, sendo os dias com LI os mais intensos. Por fim, às 18 UTC, os perfis da componente zonal não apresentam nenhuma característica marcante. Em todos os horários a componente foi predominantemente de leste.

Diferente do que foi indicado por Alcântara et al. (2011), a componente meridional parece dar sua contribuição ao processo. De forma geral, o que se percebe através dos casos analisados é que essa componente do vento sempre muda de direção com a altura passando de negativa muito próxima a superfície para positiva em torno dos $800 \mathrm{hPa}$. Este é o nível em que se observa um máximo na componente zonal ao qual chamamos de jato de baixos níveis. Nos dias com LI, esta rotação tende a ser maior que nos dias sem a formação de LI. Para o horário das 12 UTC, ele parece ser mais pronunciado que em outros horários.

Na tentativa de quantificar este cisalha- $\left.-\frac{\partial v}{\partial z}\right)$ mento em termo da componente meridional do $\left(\frac{\partial z}{\partial z}\right)$
vento, a Tabela 1 mostra a variação desta com a altura

para todos os dias em questão e em todos os horários. Este cisalhamento foi calculado entre os máximos negativo e positivo de velocidade meridional, com o intuito de quantificar o giro do vento.

Percebe-se que a maior variação da compo- nente meridional foi no dia 23/06 em que ocorreu a formação de uma linha de instabilidade bem definida e este maior valor ocorreu as 12 UTC. Uma maior $\varsigma_{x}=\left(\frac{\partial w}{\partial x}+\frac{\partial v}{\partial z}\right) \begin{aligned} & \text { variação de } \mathrm{v} \text { com a altura pode } \\ & \text { indicar um aumento na componente } \\ & \text { zonal da vorticidade já que }\end{aligned}$

e w é uma ordem de magnitude

menor que v. Esse giro pode estar contribuindo para a organização dos sistemas e até em sua propagação.

É claro que a quantidade de casos analisados aqui é insuficiente para uma conclusão consistente das informações quanto a importância da velocidade meridional, mas deixa indícios de que esta tem algum papel na formação e no desenvolvimento das linhas de instabilidade que se formam na costa norte do Brasil. Se faz necessário, uma maior quantidade de experimentos como o CHUVA e por um período maior para que sejam disponibilizados dados observacionais com alta resolução espacial e temporal. Não existe um banco de dados observacionais que nos permita estudar e entender a dinâmica de mesoescala de sistemas como as linhas de instabilidade.

\subsection{Perfis de temperaturas potencial e potencial equivalente}

As Figura 3, 4, 5 e 6 mostram os perfis de temperatura potencial $(\theta)$ e temperatura potencial equivalente $(\theta e)$ para que se possa perceber as diferenças entre os dias com e sem a formação de LI nos quatro horários em que foram lançadas as sondagens. Os perfis são razoavelmente idênticos para todos os dias, possuindo características semelhantes.

Em todos os dias a 00 UTC aparece uma camada estável estaticamente devido à camada limite noturna, bem próxima a superfície. Para as 06 UTC, a intensidade desta estabilidade estática diminui, ficando em uma camada mais profunda e atingindo $950 \mathrm{hPa}$. As 12 UTC, tem-se uma camada neutra à superfície com altura que ultrapassa os $1000 \mathrm{hPa}$. Ela corresponde a

Tabela 1 - Variação da componente meridional do vento com a altura $\left(\frac{\partial v}{\partial z}\right)[\mathrm{s}-1]$.

\begin{tabular}{|c|c|c|c|c|}
\hline Horárioldia & $20 / 06(\mathrm{sem} \mathrm{LI})$ & $23 / 06(\mathrm{com} \mathrm{LI})$ & $24 / 06(\mathrm{com} \mathrm{LI})$ & $25 / 06(\mathrm{sem} \mathrm{LI})$ \\
\hline 00 & $6,6 \times 10^{-3}$ & - & $6,7 \times 10^{-3}$ & $-5,3 \times 10^{-3}$ \\
\hline 06 & $5,9 \times 10^{-3}$ & $3,0 \times 10^{-3}$ & $5,8 \times 10^{-3}$ & $-5,3 \times 10^{-4}$ \\
\hline 12 & $6,3 \times 10^{-3}$ & $9,4 \times 10^{-3}$ & $3,2 \times 10^{-3}$ & $3,8 \times 10^{-3}$ \\
\hline 18 & $1,7 \times 10^{-3}$ & $-3,1 \times 10^{-4}$ & - & $-8,9 \times 10^{-4}$ \\
\hline
\end{tabular}




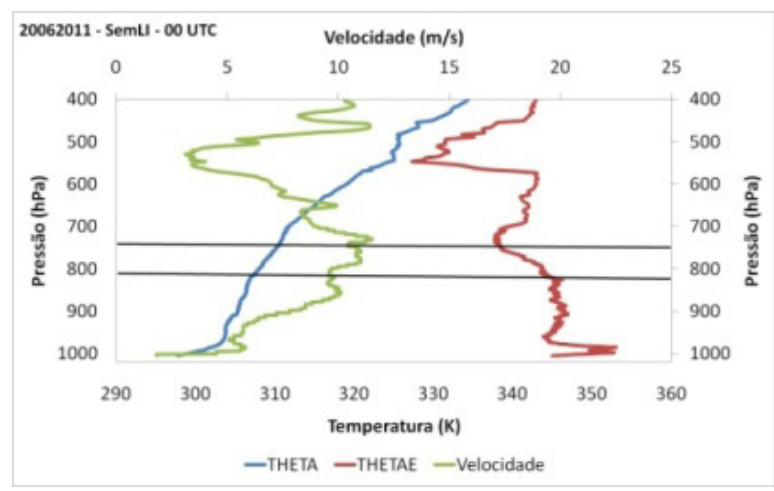

(a)

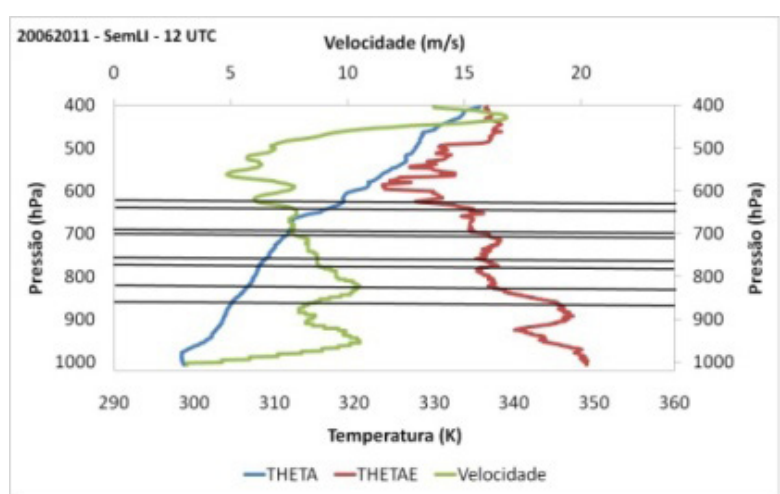

(c)

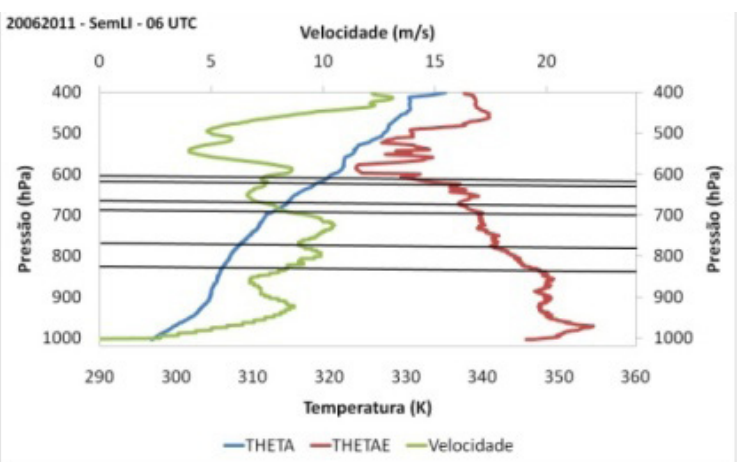

(b)

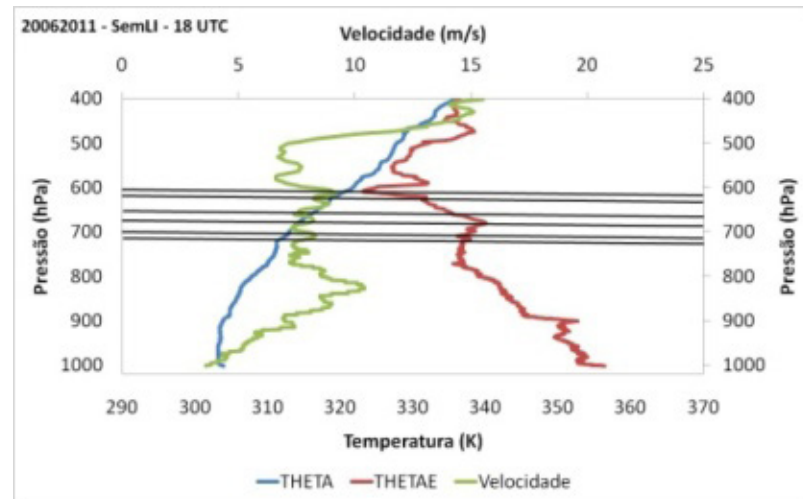

(d)

Figura 3 - Algumas camadas atmosféricas analisadas para as condições de duto em que $\left(\frac{\partial \theta_{e}}{\partial z}\right)<0$
2010 (sem LI) às 00 UTC (a), 06 UTC (b), 12 UTC (c) e 18 UTC (d). As camadas estão entre as linhas pretas.

camada de mistura que se forma devido a turbulência proveniente dos processos ligados ao aquecimento da superfície pela radiação solar. No último horário observado, as 18 UTC, esta camada neutra parece ter se tornado bastante profunda $(800-900 \mathrm{hPa})$. Este aprofundamento pode estar associado não só com a linha de instabilidade, nos dias em que se formou, mas também com a frente de brisa marítima nos dias em que não se observou a formação de LI. Ambos os sistemas são capazes de tornar mais eficientes estes processos de mistura turbulenta que ocorrem na camada limite planetária e são ótimos transportadores de momento, energia e umidade para níveis mais altos.

Quando se observa os perfis de $\theta \mathrm{e}$, percebe-se que para as 00 UTC e 06 UTC, a variação desta com a altura indica forte estabilidade potencial associada à camada limite noturna. Para as 12 UTC, os dias 20 e 23 mostraram leve instabilidade potencial e os dias 24 e 25, leve estabilidade. E as 18 UTC, todos os perfis mostraram-se instáveis potencialmente para uma camada muito próxima a superfície.

Foi discutido até aqui, apenas os níveis mais próximos à superfície a fim de se perceber se há dife- renças entre os dias com e sem formação de LI. Porém, para a análise dos dutos atmosféricos será preciso observar níveis mais altos. Logo, observando as figuras que se seguem tem-se que a atmosfera é, praticamente, estável estaticamente até $400 \mathrm{hPa}$, que é o nível disponibilizado para as análises. Esta estabilidade estática é observada em todos os dias e em todos os horários. Olhando para os perfis de $\theta$ e, percebe-se que há uma grande variação entre estabilidade e instabilidade à medida que aumenta a altura em todos os horários e em todos os dias.

Desta forma, não parece possível separar características presentes apenas em dias com LI de dias sem LI nesta análise termodinâmica. Para os dutos, ao que tudo indica, as questões termodinâmicas não tomam o papel principal. A diferenciação do ambiente de formação das LI parece estar mesmo no perfil de vento. Esta discussão será melhor desenvolvida nas seções que se seguem.

\subsection{Condições essenciais para a formação de dutos}


Inicialmente, buscou-se identificar a existência de uma camada com características próximas às descritas por LT capaz de refletir as ondas de gravidade que se propagam junto ao sistema, ou seja, uma camada refletora. Essa camada refletora deve ser potencialmente instável e deve ter número de Richardson menor que 0,25. As Figuras 3, 4, 5 e 6 mostram os perfis verticais da temperatura potencial $(\mathrm{K})$, temperatura potencial equivalente $(\mathrm{K})$ e da velocidade horizontal (componente zonal mais componente meridional) (m.s-1). Também mostram algumas dessas camadas que se apresentaram com as condições descritas acima. A Tabela 2 mostra a quantidade total de camadas por dia e horário que foram identificadas em cada perfil.

Por estes resultados, percebe-se que em todos os dias, independentemente, de haverem ou não a formação de linhas de instabilidade na costa norte do Brasil, existe as condições essenciais para a formação de uma camada refletora das ondas de gravidade que podem estar associadas com estes sistemas. Em todos os perfis, observa-se, inclusive, mais de uma camada. Através da Tabela 2, podemos observar que o horário das 06 UTC foi o período em que se observou maior existência dessas camadas, nos dias com a formação de linha de instabilidade.

Uma atmosfera com número de Richardson menor que 0,25 implica que os forçantes mecânicos, como o vento por exemplo, são quem promovem o levantamento de parcelas da atmosfera. Por estas características e por terem propriedades físicas diferentes das demais camadas abaixo desta, são capazes de impedir que as ondas de gravidade propaguem sua energia na vertical. Este "efeito tampão" faz com que a energia dessas ondas não se dissipe rapidamente e seja usada pelo sistema para propagação ou mesmo aumentar seu tempo de vida.

Porém, como vista em seções anteriores, existem outras condições que precisam ser satisfeitas para que o duto seja formado. Uma vez que camadas podem servir como refletoras das ondas de gravidade, estas

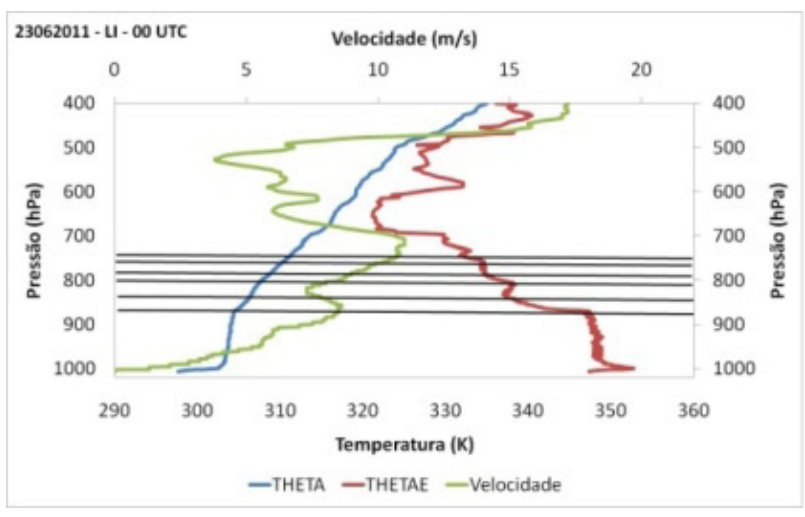

(a)

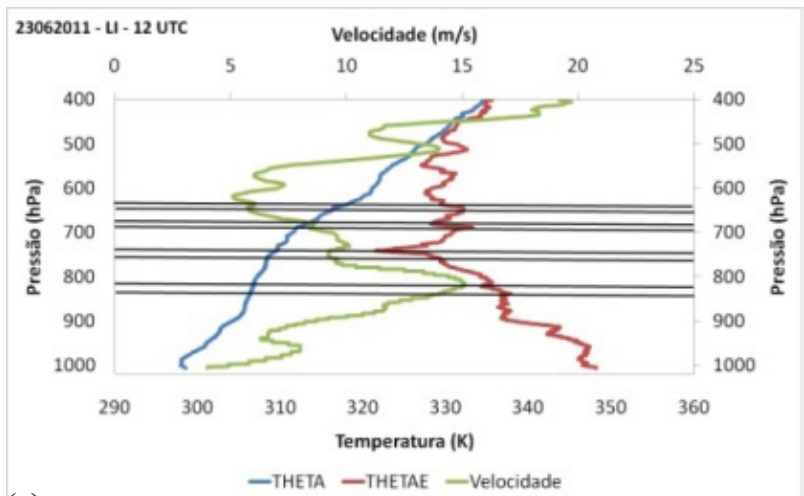

(c)

Figura 4 - Algumas camadas atmosféricas analisadas para as condições de duto em que $\left(\frac{\partial \theta_{e}}{\partial z}\right)<0$ para o dia 23 de junho de 2010 à 00 UTC (com LI) (a), 06 UTC (b), 12 UTC (c) e 18 UTC (d). As camadas estão entre as linhas pretas.

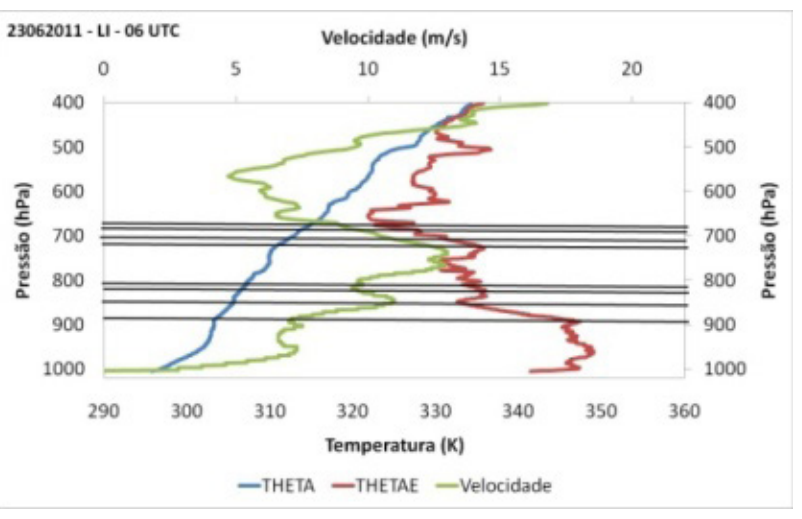

(b)

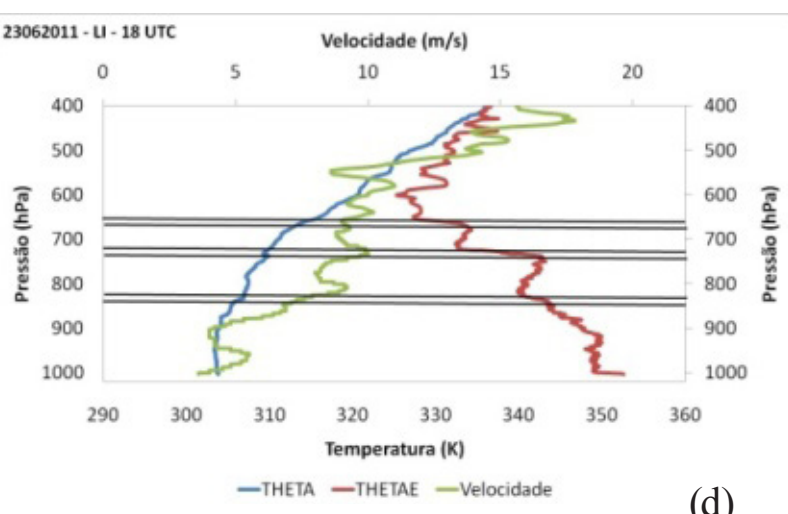

(d) 
Tabela 2 - Número de camadas que satisfazem parcialmente as condições de dutos para os dias em estudo e nos horários disponíveis (UTC). Entre parênteses é o número de camadas que possuem altura superior a $1,5 \mathrm{~km}$.

\begin{tabular}{|c|c|c|c|c|}
\hline Horárioldia & $20 / 06(\mathrm{sem}$ LI) & $23 / 06(\mathrm{com}$ LI) & $24 / 06(\mathrm{com}$ LI) & $25 / 06(\mathrm{sem}$ LI) \\
\hline 00 & $1(1)$ & $5(2)$ & $3(2)$ & 0 \\
\hline 06 & $4(4)$ & $10(9)$ & $7(6)$ & $3(3)$ \\
\hline 12 & $4(3)$ & $5(4)$ & $6(5)$ & $6(6)$ \\
\hline 18 & $3(3)$ & $3(2)$ & - & $5(5)$ \\
\hline
\end{tabular}

só conseguem se propagar em um meio estaticamente estável. Logo, abaixo das camadas encontradas potencialmente estáveis e instáveis mecanicamente, deve existir uma camada estaticamente estável. Nos casos encontrados aqui e que estão indicados nas Figuras 3 , 4,5 e 6 e na Tabela 2 , todas as camadas possivelmente refletoras possuem camadas estaticamente estáveis abaixo delas. Assim, mais uma condição é satisfeita para a existência dos dutos.

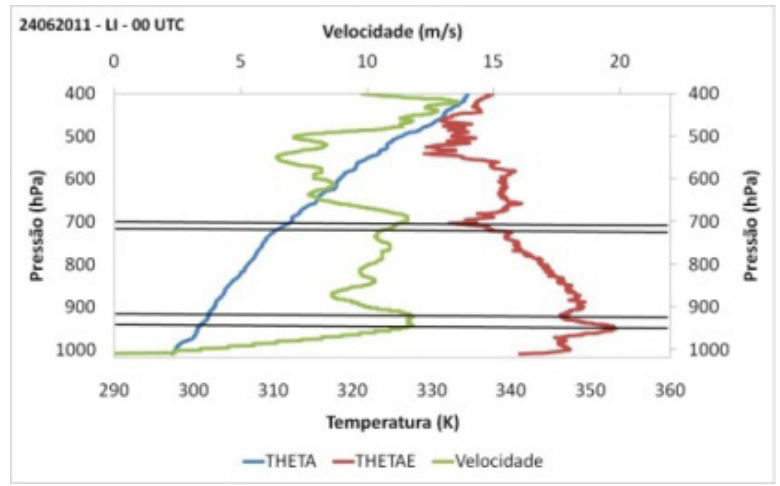

(a)

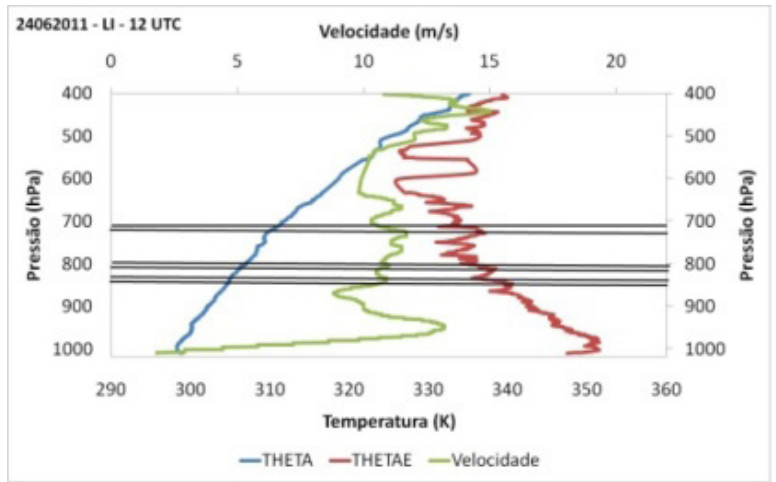

(c)

Figura 5 - Algumas camadas atmosféricas analisadas para as condições de duto em que $\left(\frac{\partial \theta_{e}}{\partial z}\right)<0$ para o dia 24 de junho de 2010 a 00 UTC (com LI) (a), 06 UTC (b), 12 UTC (c) e 18 UTC (d). As camadas estão entre as linhas pretas.
Outra condição muito importante é a espessura dessa camada abaixo da camada refletora, que é o duto propriamente dito. Ela deve ser tal que acomode, pelo menos, um quarto do comprimento vertical das ondas que estão se propagando.

Obter informações a respeito destas ondas é um trabalho complicado, pois não existem dados com resolução temporal e espacial suficientes. Mesmo com os incentivos e as várias realizações de campanhas de

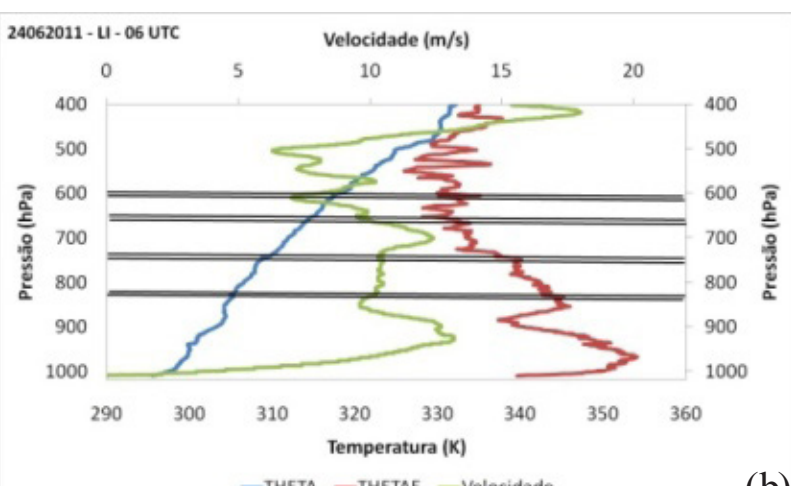

(b) 
coleta de dados realizados por toda a bacia amazônica por ocasião de vários experimentos, como por exemplo, o Large Scale Biosphere-Atmosphere Experiment in Amazonia (LBA) e o CHUVA, dados observacionais são escassos e possuem muitas descontinuidades.

Cohen (1996) verificou que as ondas associadas com as linhas de instabilidade da Amazônia tinham comprimento vertical entre 6 e $10 \mathrm{~km}$ e comprimento horizontal entre $200-250 \mathrm{~km}$. Assim, se o comprimento de onda na vertical varia entre 6 e $10 \mathrm{~km}$, isso implica que a espessura da camada de duto deve ser pelo menos igual a $1,5 \mathrm{~km}$.

Das camadas identificadas aqui, algumas não satisfazem esta condição (Tabela 2, valores entre parênteses). Apenas camadas em torno de $800 \mathrm{hPa}$ são capazes de propagar ondas de gravidade, pois estão altas o suficiente para acomodar um quarto do comprimento dessas ondas na vertical. Observa-se isto, especialmente, para o horário das 12 UTC já que, geralmente, se forma uma camada de mistura muito próxima a superfície como observado nas Figuras 3, 4, 5 e 6.

Mesmo com todas estas condições a serem satisfeitas, observa-se que mais de uma camada pode servir de camada refletora e mais de uma camada pode permitir a propagação das ondas de gravidade. Percebe-se que em termos de características termodinâmicas não é possível distinguir dias com de dias sem a formação de linhas de instabilidade.

Algumas condições ainda faltam ser observadas e todas envolvem o perfil de vento. Já foi mostrado em seções anteriores, e em trabalhos na literatura, que é possível distinguir dias de formação de linhas de instabilidade de dias sem a formação desses sistemas tanto através da componente zonal quanto da componente meridional do vento. Na seção que se segue, esta discussão será aprofundada e para facilitar o entendimento será usada a intensidade do vento com suas duas componentes somadas.

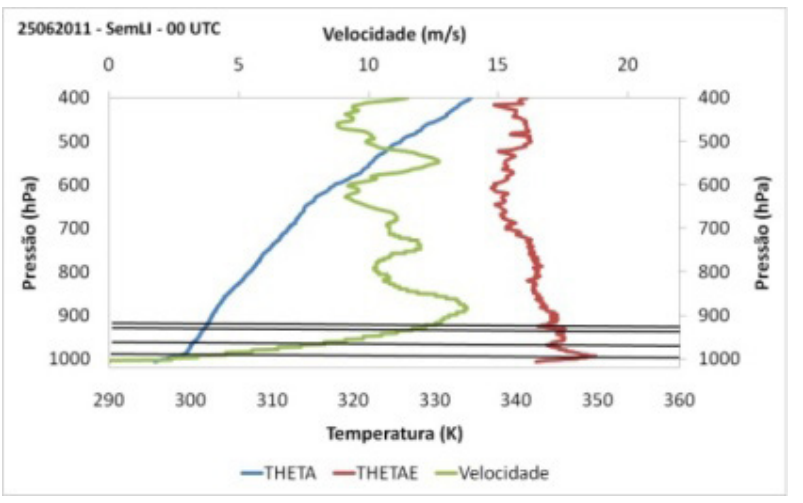

(a)

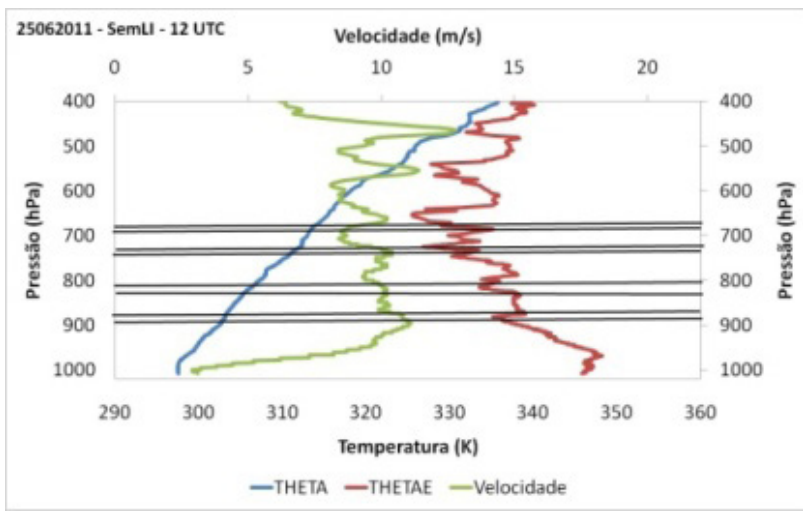

(c)

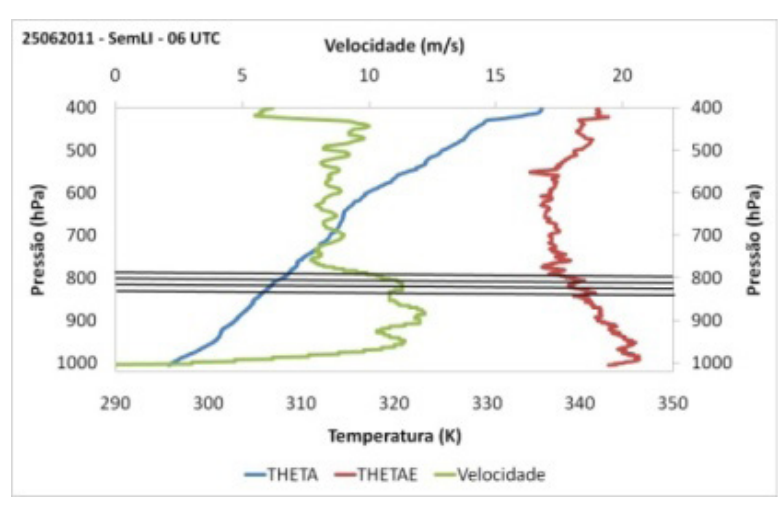

(b)

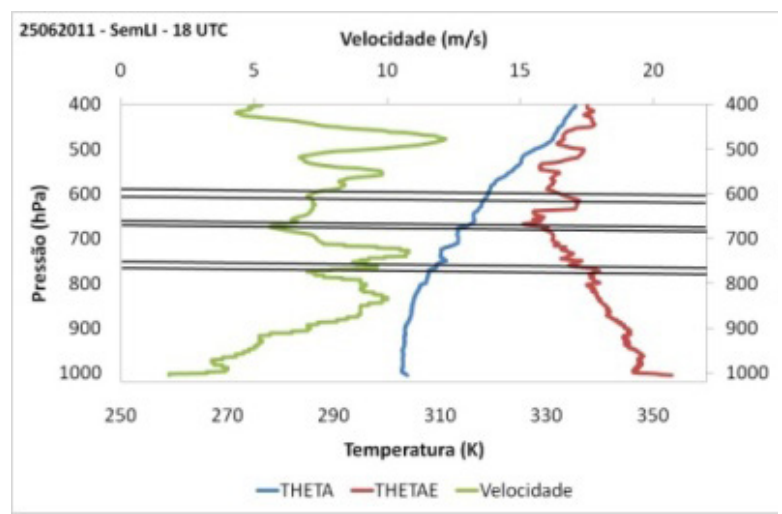

Figura 6 - Algumas camadas atmosféricas analisadas para as condições de duto em que $\left(\frac{\partial \theta_{e}}{\partial z}\right)<0$ para o dia 25 de junho de 2010 à 00 UTC (sem LI) (a), 06 UTC (b), 12 UTC (c) e 18 UTC (d). As camadas estão entre as linhas pretas. 


\subsection{Nível crítico}

Nível crítico é a camada em que a velocidade de fase das ondas de gravidade e a velocidade do escoamento se igualam. Ele é extremamente importante para a formação de dutos, pois dependendo de sua posição com relação às camadas refletora e de duto, ele pode agir para refletir e transmitir as ondas ou absorver sua energia. Se o nível crítico estiver na camada de duto, ele absorverá a energia das ondas fazendo com que não exista efetivamente este duto e o sistema tende a se dissipar muito rapidamente. Se ele estiver na camada refletora, age para ajudar na reflexão dessas ondas e no aprisionamento da energia. Se estiver logo acima da camada refletora, além de refletir as ondas, também as transmite e esta é a condição ideal observada por LT.

Aqui será feita uma estimativa da velocidade dessas ondas a partir das informações obtidas por Cohen (1996) e com o auxílio de formulações básicas demonstradas por Holton (2004) e que estão detalhadas na seção 2.2.

A Tabela 3 mostra a variação da velocidade de fase das ondas de gravidade para cada comprimento de onda na vertical e na horizontal. Percebe-se que à medida que o comprimento de onda na vertical aumenta, a velocidade das ondas também aumenta. Desta forma, elas variam entre 11 e 18 m.s-1, aproximadamente. Este é o valor tipo dos JBN (Alcântara et al., 2011; Cohen et al., 1995). Esta observação sugere que o JBN pode ser um dos elementos que contribuem com o aprisionamento das ondas de gravidade e pode explicar o fato de ele ser tão decisivo para a formação e, especialmente, na propagação das linhas de instabilidade. Nenhum dos trabalhos que associam os JBN com as LI são capazes de explicar por que existe a diferenciação entre dias com e sem formação de LI e sistemas que se propaguem mais ou menos. Apenas sabe-se que o JBN contribui para o processo, porém não se sabe, dinamicamente, qual é o seu papel.

Quando observamos os perfis de $\theta$, $\theta$ e e da velocidade do vento nas Figuras 4, 5, 6 e 7, percebe-se que os máximos de intensidade do vento, ou seja os
JBN, são em torno dos 10 - 15 m.s-1. Esses máximos relativos, ora aparecem em níveis muito mais baixos, ora parecem em níveis um pouco mais elevados. Isto pode fazer toda a diferença em termos dos dutos atmosféricos, pois pode indicar o nível de ocorrência do nível crítico.

Então, parte das condições para existência de dutos atmosféricos foram encontradas nos casos de linha de instabilidade escolhidas aqui. Este mecanismo poderia ser responsável pela propagação destes sistemas por longas distâncias. Em trabalhos futuros, pretende-se averiguar quais são as características das ondas de gravidade associadas as linhas de instabilidade da Amazônia e, assim, aplicar adequadamente a teoria dos dutos atmosféricos a uma quantidade significativa de casos.

\section{Conclusão}

Aqui foi proposto que a teoria dos dutos atmosféricos descrita por Lindzen e Tung (1976) poderia explicar esta propagação já que outros autores constataram a existência de ondas de gravidade se propagando junto a estes sistemas, mas apenas sugerira a existência de dutos. Assim, não existe na literatura atual um trabalho que mostre sistematicamente, muito menos utilizando dos observados, a presença de dutos associados com as linhas de instabilidade da Amazônia.

A aplicação das propriedades dos dutos atmosféricos a casos de LI mostrou que estas foram satisfeitas em sua maioria, indicando que talvez estes possam explicar sua propagação e que em alguns casos se propaguem por milhares de quilômetros. O que fica mais evidente nesta análise preliminar, é que os jatos de baixos níveis, observados constantemente nos perfis de vento associados com as LI, tem um papel de grande destaque uma vez que podem indicar níveis críticos onde a energia das ondas de gravidade está sendo aprisionada.

As próximas etapas da pesquisa tentaram elucidar as seguintes questões: estas propriedades de

Tabela 3 - Variação da velocidade de fase de ondas de gravidade com comprimento horizontal entre 200 e $250 \mathrm{~km}$ e comprimento vertical entre 6 e $10 \mathrm{~km}$.

\begin{tabular}{|c|c|c|c|c|c|c|}
\hline $\mathbf{m} / \mathbf{k}$ & $\mathbf{2 \pi / 2 0 0}$ & $\mathbf{2 \pi / 2 1 0}$ & $\mathbf{2 \pi / 2 2 0}$ & $\mathbf{2 \pi / 2 3 0}$ & $\mathbf{2 \pi / 2 4 0}$ & $\mathbf{2 \pi / 2 5 0}$ \\
\hline $\mathbf{2 \pi / 1 0}$ & 18,42 & 18,42 & 18,43 & 18,43 & 18,43 & 18,43 \\
\hline $\mathbf{2} \boldsymbol{\pi} / \mathbf{9}$ & 16,58 & 16,59 & 16,59 & 16,59 & 16,59 & 16,59 \\
\hline $\mathbf{2} \boldsymbol{\pi} / \mathbf{8}$ & 14,74 & 14,75 & 14,75 & 14,75 & 14,75 & 14,75 \\
\hline $\mathbf{2} \boldsymbol{\pi} / \mathbf{7}$ & 12,90 & 12,90 & 12,91 & 12,91 & 12,91 & 12,91 \\
\hline $\mathbf{2} \boldsymbol{\pi} \mathbf{6}$ & 11,06 & 11,06 & 11,06 & 11,06 & 11,06 & 11,06 \\
\hline
\end{tabular}


dutos são observadas em outros casos? Qual o padrão destas ocorrências de dutos?

\section{Referências}

ALCÂNTARA, C. R.; SILVA DIAS, M. A. F.; SOUZA, E. P.; COHEN, J. C. P.; 2011: Verification of the Role of the Low Level Jets in Amazon Squall Lines. Atmospheric Research, v. 100, 36-44.

Battaglia, A.; Saavedra, P.; Morales, C. A.; Simmer, C.; 2011. Understanding three dimensional effects in polarized observations with the ground based ADMIRARI radiometer during the CHUVA campaign. Journal of Geophysical Research, vol. 116, D09204, 1-17.

Chun, h.-y; lin y.-1; 1995. Enhanced response of an atmospheric flow to a line-type heat sink in the presence of a critical level. Meterol. Atmos. Phys., v. $55,33-45$.

COHEN, J. C. P.; SILVA DIAS, M. A. F.; NOBRE, C. A.; 1995: Environmental conditions associated with amazonian squall lines: a case study. Mon. Wea. Rev. 123, 3163-3174

COHEN (1996). Propagation mechanism and Amazon squall line structure (in Portuguese). $\mathrm{PhD}$ dissertation, University of São Paulo, São Paulo Brazil.

HOLTON, J. R.; 2004: An introduction to dynamic meteorology, fourth edition. Elsevier Academic Press, Estados Unidos da América.

KOUSKY, V. E.; 1980: Diurnal rainfall variation in the northeast Brazil. Mon. Wea. Rev., 108, 488-498.

LINDZEN, R.S., K. -K. TUNG (1976). Banded convective activity and ducted gravity waves, Mon. Wea. Rev., 104, 1602-1617.

Monserrat, s.; Thorpe, a. j.; 1996. Use of ducting theory in an observed case of gravity waves. J. atmos. Scie., vol. 53, 1724-1736.

Ralph, F. m.; crochet, m.; venkateswaran, v.; 1993. Observations of a mesoscale ducted gravity wave. J. Atmos. Scie., v. 50, 3277-3291.

SESAME; 1974: Project Development Plan. NOAA Environmetal Resarch Laboratories, Boluder, Colo.
SILVA DIAS, M. A. F.; FERREIRA, R. N.; 1992: Application of linear spectral model to the study of amazonian squall lines during GTE/ABLE 2B. J. Geophy. Res., 97, 20405-20419.

Souza, E. P.; Leitão, M. M. V. B. R.; Barbosa, T. F.; 2001. Características da atmosfera superior, a partir de dados de alta resolução obtidos à superfície. Revista Brasileira de Engenharia Agrícola e Ambiental, v.5, n.3, 463-468.

sun, w. y.; Orslanski, I.; 1981: Large mesoscale convection and sea breeze circulation. Part I: Linear stability analysis. J. Atmos. Sci., 38, 16751693.

WARNER, T. T.; MAPES, B. E.; XU, M.; 2003. Diurnal patterns of rainfall in northwestern south America. Part II: model simulations. Mon. Wea. Rev., v. 131, 813-829. 